\title{
Successful treatment of Charles Bonnet Syndrome with sodium valproate and sertraline: A case report \\ DVM Dias, C Suraweera
}

\section{Abstract}

Charles Bonnet Syndrome (CBS) is associated with varying degrees of distress and impairment. However there is no universally accepted or established treatment to manage this condition. We report a patient who had CBS secondary to severe glaucoma and cataract, leading to severe depression, who responded well to treatment with sodium valproate and sertraline.

Key words: Charles Bonnet Syndrome, visual hallucinations, sodium valproate, sertraline

SL J Psychiatry 2020; 11(2): 31-32

\section{Introduction}

Charles Bonnet Syndrome (CBS) refers to complex visual hallucinations experienced by people with either partial or complete visual impairment, who are otherwise psychologically normal. The condition is named after Charles Bonnet, who in 1760 documented the vivid and complex visual hallucinations experienced by his 87-yearold grandfather, who had a severe visual impairment (1). This condition is reported in $11-14 \%$ of people with visual impairment (1).

\section{Case Report}

Mrs. S is a 61-year-old female, who was referred to the liaison psychiatry unit by a Consultant Ophthalmologist, in the context of her experiencing persistent, clear and vivid visual hallucinations for the last five years. She had undergone intensive therapy for glaucoma and had undergone surgery for bilateral cataracts. However her best corrected vision remained at 06/60.

Her visual hallucinations included clear images of her face and that of other people known to her. These appeared inside colourful boxes and tended to move quickly. She also experienced her body movements in the form of visual hallucinations, which occurred rarely. She reported that these hallucinations were present continuously and did not disappear even when she closed her eyes. This had resulted in difficulties in falling asleep and impairment of her activities of daily living. The hallucinations had worsened in their intensity over the previous one year, and she had subsequently developed depressive symptoms with ideas of suicide. She did not have any other psychotic symptoms. Her
Montreal cognitive assessment (MoCA) was within normal limits. All investigations including non-contrast CT scan of the brain were normal.

Behavioural strategies such as closing her eyes and repetitive blinking and distraction failed to alleviate her hallucinations or the distress caused by them. She was started on risperidone $0.5 \mathrm{mg}$ and sertraline $25 \mathrm{mg}$ daily. She developed significant extrapyramidal side effects when the risperidone dose was increased to $1 \mathrm{mg}$, and therefore this treatment was ceased. Her depressive symptoms responded to a dose of sertraline to $50 \mathrm{mg}$, but she continued to experience visual hallucinations. Therefore, she was prescribed sodium valproate $200 \mathrm{mg}$ twice a day. After continuing the combination of sertraline $50 \mathrm{mg}$ daily and sodium valproate $200 \mathrm{mg}$ twice a day for two months, she reported complete resolution of visual hallucinations. She had resumed her activities of daily living without much support.

\section{Discussion}

The following diagnostic criteria have been proposed by Teunisse et. al., to diagnose CBS (2).

a) At least one complex visual hallucination within the past four weeks

b) A period between the first and the last hallucination exceeding four weeks

c) Full or partial retention of insight into the unreal nature of the hallucinations

d) Absence of hallucinations in other sensory modalities and delusions

e) The hallucinations cannot be explained by the presence of another psychiatric disorder. 
The hallucinations in CBS occur in clear consciousness and are exclusively visual, and coexist with normal perceptions (1). They range from dots and lines to inanimate objects, scenes, animals and humans $(3,4)$. Most often these hallucinations are of human faces or figures (1). Autoscopic hallucinations have also been described, as in our patient (1). The size of the hallucination could be miniature, normal-sized or "larger than life" (1). Most patients have experienced the hallucinations while their eyes are open, but in others, as in the case of Mrs. S, the hallucinations are experienced even with their eyes closed $(1,4)$. In most cases, the hallucinations do not have any personal significance and do not affect general well-being (4). However, as in the case of our patient, there are reports of patients who have experienced distress and fear of insanity secondary to the experience of hallucinations (1). There are also reports of these hallucinations being precipitated by sensory reduction, stress, fatigue, low levels of illumination, and bright light (1). In our patient, fatigue and emotional stress were reported as triggers.

\section{Pathophysiology}

CBS is generally associated with low visual acuity and advanced age, but can occur with normal visual acuity and in patients with visual field defects $(2,5)$. Our patient had severe, treatment-refractory visual impairment. Although the exact mechanism of CBS is unclear, the most widely accepted theory is the de-afferentation theory, which describes that hallucinations occur due to spontaneous neuronal discharge, secondary to a diminution of visual sensory input (1).

\section{Treatment}

Benign cases may not need any treatment (1). Specific treatment is indicated if the hallucinations are frequent, persisting, distressing, or cause impairment of quality of life (1). The hallucinations usually disappear with correction of visual impairment or with complete blindness, and maximizing of visual functions has been reported as the first step of management $(1,6)$. However, the visual impairment of Mrs. S remained refractory to several modes of treatment. Reassurance, education and simple behavioural techniques have been shown to help in the amelioration of the hallucinations in some cases, while cognitive restructuring, relaxation, hypnosis and modifying the environment may also be effective $(1,4,7)$. Reassurance reduced the distress in our patient, but she found it difficult to engage in the behavioural techniques, due to the intensity of the hallucinations.

Currently, there are no effective pharmacological treatments for CBS. According to case reports, neuroleptics including olanzapine, thioridazine, haloperidol, risperidone, melperone and pimavanserin have been used with some success (1). We prescribed sertraline mainly for the patient's depression. Antidepressants such as SSRI, venlafaxine and mirtazapine have been successful in treating CBS. In our patient too, sertraline appears to have contributed to the improvement of symptoms.

Antiepileptics, such as carbamazepine, clonazepam, valproate, pregabalin and gabapentin have also been used effectively, and donepezil and cisapride have also been reported to improve symptoms $(1,3)$. Mrs.S showed a drastic reduction in her hallucinations after the sodium valproate was added to her prescription regimen.

\section{Conclusion}

Similar to previous case reports, this care report highlights that the prescription of sodium valproate and sertraline can be an effective treatment for CBS.

\section{Statement of contribution}

Both authors contributed equally to the writing of the case report.

\section{Conflicts of interest}

None declared.

DVM Dias, C Suraweera, University Psychiatry Unit, National Hospital of Sri Lanka.

Corresponding author: DVM Dias

Email: madhu640@gmail.com

http://orcid.org/0000-0002-2729-178X

\section{References}

1. Menon GJ, Rahman I, Menon SJ, Dutton GN. Complex Visual Hallucinations in the Visually Impaired: The Charles Bonnet Syndrome. Surv Ophthalmol 2003; 48(1): 58-72.

2. Teunisse RJ, Cruysberg JRM, Verbeek A, Zitman FG. The Charles Bonnet Syndrome: A Large Prospective Study in the Netherlands: A Study of the Prevalence of the Charles Bonnet Syndrome and Associated Factors in 500 Patients Attending the University Department of Ophthalmology at Nijmegen. BJPsych 1995; 166(2): 254-7.

3. Brucki S, Takada L, Nitrini R. Charles Bonnet Syndrome: Case series. Dement Neuropsychol 2009; 3(1): 61-7.

4. Teunisse RJ, Zitman FG, Cruysberg JRM, Hoefnagels WHL, Verbeek ALM. Visual hallucinations in psychologically normal people: Charles Bonnet's syndrome. Lancet. 1996; 347(9004): 794-7.

5. Freiman TM, Surges R, Vougioukas VI, Hubbe U, Talazko $\mathrm{J}$, Zentner J, et al. Complex visual hallucinations (Charles Bonnet syndrome) in visual field defects following cerebral surgery. Report of four cases. J Neurosurg 2004; 101(5): 846-53.

6. Eperjesi F, Akbarali N. Rehabilitation in Charles Bonnet syndrome: a review of treatment options. Clin Exp Optom. 2004; 87(3): 149-52.

7. Issa BA, Yussuf AD. Charles bonnet syndrome, management with simple behavioral technique. J Neurosci Rural Pract. 2013; 4(1): 63-5. 